Ann. Zootech., I97I, $20(4), 477-486$.

\title{
INFLUENCE DU POIDS VIF ET DU NIVEAU D'INGESTION SUR LE COMPORTEMENT ALIMENTAIRE ET MERYCIQUE DU MOUTON
}

\author{
J.-P. DULPHY \\ avec la collaboration technique de J.-M. Borsseau \\ Station de Recherches sur l'Elevage des Ruminants, \\ Centre de Recherches de Clermont-Ferrand, I. N.R. A., \\ 63 - Saint-Genès-Champanelle
}

\section{RÉSUMÉ}

I. L'influence du poids vif et du niveau d'ingestion sur les manifestations du comportement alimentaire et mérycique a été étudiée sur 17 moutons recevant pendant 6 jours le même fourrage vert (deuxième cycle de brome).

2. Il ressort de cette étude que le poids vif des animaux n'a pas d'action sur les durées journalières d'ingestion et de rumination. Par contre, lorsque le poids vif augmente les quantités de matière sèche ingérées (en $\mathrm{g} /$ jour) augmentent et les durées unitaires d'ingestion et de rumination (en minutes par $\mathrm{kg}$ de matière sèche ingérée) diminuent. En revanche les quantités ingérées exprimées en $\mathrm{g} / \mathrm{kg} \mathrm{P}^{\mathbf{0}, 75}$ et les durées unitaires exprimées en minutes par $\mathrm{g}$ ingéré par $\mathrm{kg} \mathrm{P}^{\mathbf{0 , 7 5}}$ sont indépendantes du poids vif des animaux.

3. D'un mouton à l'autre la quantité ingérée (en $\mathrm{g} / \mathrm{kg} \mathrm{P}^{\mathbf{0}, 75}$ ) $\epsilon$ st en liaison positive avec la durée journalière de rumination et en liaison négative avec les durées unitaires d'ingestion et de rumination; dans ces conditions la durée journalière d'ingestion ne varie pas.

4. D'un jour à l'autre lorsque la quantité ingérée par un mouton augmente les durées journalières d'ingestion et de rumination augmentent tandis que les durées unitaires d'ingestion et de rumination diminuent.

5. Les relations entre le comportement des animaux et les quantités qu'ils ingèrent sont discutées. Ainsi la durée journalière d'ingestion semble n'être qu'une conséquence de la quantité ingérée. Par contre, il est difficile de dire si les variations de la durée unitaire d'ingestion sont la cause ou la conséquence des variations des quantités ingérées. Enfin dans cette expérience la rumination apparaît plus comme un phénomène passif influencé par les quantités ingérées.

\section{INTRODUC'TION}

L'enregistrement graphique des mouvements de la mâchoire inférieure permet de mesurer, de façon simple et précise, les manifestations du comportement alimentaire des ruminants : durée, vitesse et répartition en 24 heures de l'ingestion et de la rumination. 
Les manifestations du comportement alimentaire étant en liaison avec les quantités ingérées (GoRdon, I965; WELCH et SMrTH, I969; KERBAA, I969; et autres), une comparaison précise des réactions des animaux recevant des fourrages de qualité ou de nature différentes peut être une des méthodes d'étude des phénomènes buccaux et digestifs intervenant dans la régulation de l'appétit et peut permettre de mieux interpréter les variations des quantités ingérées entre fourrages. C'est la raison pour laquelle, dans le cadre de l'étude entreprise à la Station sur la valeur alimentaire des fourrages verts et conservés (DEMARQUILLY, WEISS, I970) les manifestations du comportement alimentaire des moutons sont enregistrées. Or les moutons utilisés pour cette étude sont souvent de poids différents suivant les essais, et leur poids varie au cours des essais de longue durée. Ayant commencé le dépouillement de ces mesures de comportement alimentaire, il nous a semblé que certains des paramètres comportementaux, notamment les vitesses d'ingestion et de rumination, exprimées par beaucoup d'auteurs en temps passé à ingérer ou à ruminer par $\mathrm{kg}$ de matière sèche ingérée semblaient dépendre du poids de l'animal. De même, Gordon (I969) observe des différences considérables dans le nombre de coups de mâchoire et l'efficacité de la mastication pendant la rumination et note qu'elles seraient peut-être liées au poids des animaux. Il est donc nécessaire d'exprimer les paramètres comportementaux dans une unité indépendante du poids de l'animal.

Récemment, WELCH et al (I969, I970), pour comparer l'efficacité de la rumination de différents moutons, a proposé de corriger le temps de rumination en tenant compte du poids métabolique des animaux. Cela est-il justifié, et dans 1'affirmative, la correction doit-elle aussi s'appliquer au temps d'ingestion? C'est ce que nous avons voulu étudier en enregistrant les manifestations du comportement alimentaire de $\mathrm{I} 7$ moutons de poids très différents (40 à $85 \mathrm{~kg}$ ) qui recevaient un même fourrage vert. Cette étude devrait en outre nous permettre d'évaluer l'importance des variations individuelles entre animaux.

\section{MATÉRIEL E'T MÉ'THODES}

Nous avons choisi, dans le troupeau au pâturage, 17 moutons mâles, castrés, de race Texel. Leurs poids se répartissaient en moyenne tous les $5 \mathrm{~kg}$ entre 40 et $85 \mathrm{~kg}$. 6 moutons étaient âgés de $\mathrm{I}$ an, 5 de 2 ans, 5 de 3 ans $\epsilon t$ I de 5 ans. Ces moutons ont été maintenus en cage à métabolisme et y ont reçu un fourrage vert : deuxième cycle de brome (Bromus inermis). Ce fourrage vert, fauché chaqua matin à 9 heures, était distribué à volonté ( 10 p. Ioo de refus) en 3 repas, à I I h 30, I 7 heures et à 8 heures le lendemain ; les refus étaient enlevés avant la distribution du repas de 8 heures. Après une période d'adaptation de io jours au fourrage étudié, la quantité journalière cle matière sèche a été mesurée individuellement pendant 6 jours par pesée des quantités distribuées et refusées et par détermination des teneurs en matière sèche de l'herbe offerte à chaque repas et des refus journaliers. Pendant ces 6 jours, le comportement alimentaire de chaque mouton a été mesuré de façon continue par l'enregistrement des mouvements de la mâchoire selon la méthode de RuckEBusch ( 1963$)$. Les durées journalières d'ingestion et de rumination ont été mesurées sur les bandes d'enregistrement et exprimées en minutes.

Durant la période expérimentale de 6 jours, la digestibilité 'de la matière organique de l'herbe distribuée a été de 69,2 p. Ioo, sa teneur en matière sèche a augmenté régulièrement de 26, I à 29,5 p. I oo, exception faite pour 3 repas d'une baisse à 23,9 p. Ioo consécutive à une pluie survenue au milieu de la période.

Les moutons ont été pesés au début et à la fin de la période expérimentale. 


\section{RÉSULTATS}

\section{Quantités ingérées (QI)}

Les quantités ingérées ont été très variables d'un animal à l'autre (tabl. I) : nous assimilerons ces variations à des variations d'appétit. Pour un mouton donné, elles ont en outre varié d'un jour à l'autre ; les plus faibles ont été enregistrées quand l'herbe distribuée avait été mouillée par la pluie.

La quantité journalière moyenne de matière sèche ( $y$ en $g$ ) ingérée par mouton a

TABI,EAU I

Résultats

\begin{tabular}{|c|c|c|c|}
\hline & $n$ & Moyenne & $\begin{array}{l}\text { Coefficient } \\
\text { de variation } \\
\text { p. } 100\end{array}$ \\
\hline Quantités ingérées: & & & \\
\hline & 17 & $\begin{array}{l}1217 \pm 319 \mathrm{~g} \\
\left(78^{\prime} \mathrm{à} 1924 \mathrm{~g}\right)\end{array}$ & \\
\hline en $g / k g P^{0,75} \ldots \ldots \ldots$ & 17 & $55,3 \pm 5,9 \mathrm{~g}$ & 10,7 \\
\hline Durée journalière d'ingestion ....... & 17 & $\begin{array}{c}273 \pm 26,4 \mathrm{mn} \\
(19,0 \text { p. } 100 \text { du temps journalier })\end{array}$ & 9,7 \\
\hline $\begin{array}{l}\text { Durée unitaire d'ingestion } \ldots \\
\qquad \mathrm{mn} / \mathrm{kg} \text { MS }\end{array}$ & 17 & $\begin{array}{l}238 \pm 50,5 \mathrm{mn} / \mathrm{kg} \text { MS } \\
(143 \text { à } 327 \mathrm{mn} / \mathrm{kg} \mathrm{MS})\end{array}$ & 21,2 \\
\hline $\begin{array}{l}\text { Durée unitaire d'ingestion } \\
\mathrm{mn} / \mathrm{g} / \mathrm{kg} \mathbf{P}^{0,75}\end{array}$ & 17 & $4,99 \pm 0,53$ & 10,6 \\
\hline Durée journalière de rumirıtion .. & 17 & $\begin{array}{c}537 \pm 34 \mathrm{mn} \\
(37,2 \text { p. } 100 \mathrm{du} \text { temps journalier })\end{array}$ & 6,3 \\
\hline $\begin{array}{l}\text { Durée unitaire de rumination } \\
\qquad \mathrm{mn} / \mathrm{kg} \mathrm{MS}\end{array}$ & 17 & $\begin{array}{c}4_{4} 68 \pm 97 \mathrm{mn} / \mathrm{kg} \mathrm{MS} \\
(351 \text { à } 679)\end{array}$ & 20,7 \\
\hline $\begin{array}{l}\text { Durée unitaire de rumination } \\
\mathrm{mn} / \mathrm{g} / \mathrm{kg} \mathrm{P} \mathrm{P}^{0,75}\end{array}$ & 17 & 9,76 土 0,55 & 5,6 \\
\hline Durée jourualière de mastication & 17 & $\begin{array}{c}809 \pm 44 \mathrm{mn} \\
(56,2 \text { p. } 100 \mathrm{du} \text { temps journalier })\end{array}$ & 5,4 \\
\hline $\begin{array}{l}\text { Durée unitaire de mastication } \ldots . . . \\
\mathrm{mn} / \mathrm{kg} \text { MS }\end{array}$ & 17 & $\begin{array}{c}706 \text { 上 } 142 \mathrm{mn} / \mathrm{kg} \text { MS } \\
(513 \text { à } 1005)\end{array}$ & 20,1 \\
\hline $\begin{array}{l}\text { Durée unitaire de mastication } \\
\mathrm{mn} / \mathrm{g} / \mathrm{kg} \mathrm{P}^{0,75}\end{array}$ & 17 & $14,75 \pm 0,92$ & 6,2 \\
\hline
\end{tabular}


été liée aut poids ( $\mathrm{P}$ en $\mathrm{kg}$ ) par la relation : $y=\mathrm{I} 6 \mathrm{I}+\mathrm{I7,2} \mathrm{P}\left(r=0,89^{* * *}\right)$. Cette relation indique que la quantité ingérée rapportée au poids vif est d'autant plus faible que l'animal est plus lourd: $\frac{\mathrm{Y}}{\mathrm{P}}=\mathrm{I} 7,2+\frac{\mathrm{I} 6 \mathrm{I}}{\mathrm{P}} \cdot$ Nous avons donc cherché quel exposant $(x)$ du poids il fallait prendre pour que le rapport $\frac{\mathrm{Y}}{\mathrm{P}}$ soit constant, c'est-à-dire indépendant du poids de l'animal. Cet exposant est égal à $0,89\left(r=0,90^{* *}\right)$ pour les I7 moutons, mais seulement à $0,79\left(r=0,8 \mathrm{I}^{* *}\right)$ pour les I3 moutons d'un poids supérieur à $50 \mathrm{~kg}$ ou pour les II moutons âgés de plus d'un an. Ces valeurs ne sont cependant pas significativement différentes de l'exposant 0,75 habituellement retenu. Nous exprimerons donc les quantités ingérées en $g$ par $\mathrm{kg} \mathrm{P}^{\mathrm{j}, 75}$ (tab1. I) ; cette méthode réduit d'ailleurs considérablement le coefficient de variation des résultats $(26,2$ p. Ioo pour $y$ contre I0, 7 p. IOO pour $\left.\frac{y}{\mathrm{P}^{3,75}}\right)$.

L'analyse de la variance des résultats ainsi exprimés montre que la quantité ingérée a été significativement plus faible durant les 24 heures où les moutons ont reçu l'herbe mouillée par la pluie et qu'il demeure des différences significatives entre moutons.

\section{Durée journalière d'ingestion (DI)}

La durée journalière d'ingestion a varié selon les jours et selon les moutons (effets hautement significatifs, cf. tabl. 2).

\section{TABLEAU 2}

Résultats des analyses de variance

A. - Quantités ingérées par jour en g/kg $\mathrm{P}^{0,75}$

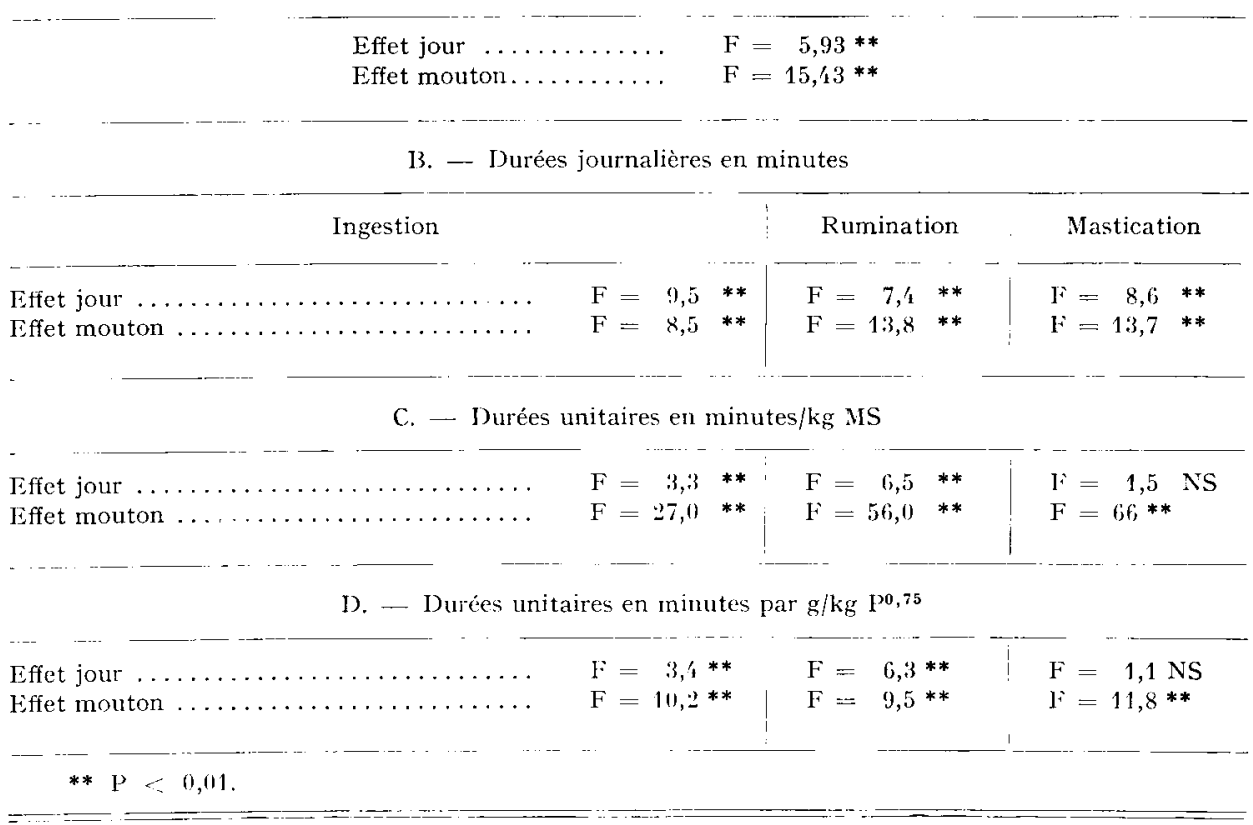


Plus précisément, il existe une liaison étroite entre cette durée journalière d’ingestion (en minutes) pour l'ensemble des moutons et la quantité journalière moyenne ingérée correspondante qui varie de I I23 à I $233 \mathrm{~g} / \mathrm{j}$.

$$
\mathrm{DI}=-\mathrm{I} 5^{\circ}+0,353 x\left(r=0,84^{*} ; n=6 \text { jours }\right)
$$

Cette liaison est encore plus nette $\left(r=0,95^{*}\right)$ si on classe pour les I 7 moutons les données par niveau d'ingestion (six niveaux pour un mouton) croissant et si on retient pour chaque niveau la valeur moyenne de l'ensemble (moyenne de I 085 à I 3 I6 g).

Le temps passé par un mouton à ingérer un fourrage donné et la quantité qu'il ingère varient donc dans le même sens.

Par contre, nous n'avons pu mettre en évidence une liaison de la durée d'ingestion, ni avec le poids $(r=0,36$, NS), ni avec les variations d'appétit d'un animal à l'autre $(r=0,20, \mathrm{NS})$ ni avec l'âge. D'un mouton à l'autre, ce sont donc des caractéristiques individuelles autres que le poids, l'appétit et l'âge qui doivent déterminer le temps passé à ingérer un fourrage donné.

\section{Durée unitaire d'ingestion (DUI)}

La durée unitaire d'ingestion est habituellement mesurée en rapportant le temps passé à ingérer à la quantité de matière sèche ingérée exprimée en $\mathrm{kg}$. Les variations de ce paramètre d'un mouton à l'autre et d'un jour à l'autre sont hautement significatives (tab1. I et 2 ).

Pour un même mouton, la durée unitaire d'ingestion diminue lorsque les quantités qu'il ingère augmentent $(x$ en $\mathrm{g} / \mathrm{j})$ :

$$
\left(r=-0,86^{*}\right) \quad \text { (cf. tabl. 3) }
$$

Entre moutons, ce paramètre diminue également lorsque l'appétit augmente $\left(r=-0,66^{* *} ; n=\mathrm{I} 7\right)$ et surtout lorsque le poids vif augmente $\left(r=-0,82^{* *}\right.$; $n=\mathrm{I} 7$ ).

Or le poids vif ayant lui-même une action sur les quantités ingérées en $g / j$, on peut se demander s'il ne faut pas exprimer la durée unitaire d'ingestion en temps passé à ingérer $\mathrm{I} g$ par $\mathrm{kg}$ de poids métabolique. C'est d'ailleurs ce qu'a fait WELCH (I970) pour exprimer la durée unitaire de rumination.

Exprimée de cette manière, la durée unitaire d'ingestion devient indépendante du poids vif de l'animal ( $r=0,0 I 6, \mathrm{NS}$ ). Cela permet de comparer entre eux des moutons de poids ou d'âges différents puisqu'elle a été identique pour les 6 moutons d'un an et les II moutons de 2 ans ou plus. Ce paramètre reste cependant lié à l'appétit des animaux : $\left(x\right.$ en $\left.\mathrm{g} / \mathrm{kg} \mathrm{P}^{0,75}\right)\left(r=-0,59^{*}\right)$ et peut varier significativement d'un jout à l'autre pour le même animal.

\section{Durée journalière de rumination (DR)}

La durée moyenne journalière de rumination a été significativement inférieure le jour où il a plu et le dernier jour de mesure. Il n'existe pas de liaison significative $(r=0,5 \mathrm{r} ; n=6)$ entre cette durée journalière moyenne pour les I7 moutons et la quantité ingérée moyenne correspondante ( $x$ en $\mathrm{g} / \mathrm{j}$ ). Par contre, pour un même mouton, la durée de rumination augmente lorsque les quantités qu'il ingère augmentent: $\left(r=0,93^{* *}\right)$. 
D'un animal à 1'autre la durée journalière de rumination a été indépendante du poids vif $(r=0,36, \mathrm{NS})$ et de l'âge des animaux, mais a été d'autant plus élevée que l'appétit augmentait $(r=0,69 * *)$.

TABLEAU 3

Facteurs de variations des paramètres $d u$ comportement alimentaire

\begin{tabular}{|c|c|c|c|}
\hline & Poids vif & $\begin{array}{c}\text { Niveau d'ingestion } \\
\text { inter-mouton }(n=17)\end{array}$ & $\begin{array}{l}\text { Niveau d'ingestion } \\
\text { intra-mouton }(n=6)\end{array}$ \\
\hline $\begin{array}{c}\text { Durée d'ingestion } \\
\text { par jour }\end{array}$ & Pas de liaison & Pas de liaison & $\begin{array}{c}+3,27 \mathrm{mn} / \mathrm{g} / \mathrm{kg} \mathrm{P} \mathrm{P}^{0,75} \\
+0,152 \mathrm{mn} / \mathrm{g} \text { ingéré } \\
r=0,95^{* *}\end{array}$ \\
\hline $\begin{array}{l}\text { Durée d'ingestion } \\
\text { par kg MS }\end{array}$ & $\begin{array}{c}-2,7 \mathrm{mn} / \mathrm{kg} \mathrm{MS} \\
\operatorname{par} \mathrm{kg} \mathrm{PV} \\
r=-0,82 * *\end{array}$ & $\begin{array}{r}-5,6 \mathrm{mn} / \mathrm{kg} \mathrm{MS} \\
\text { par } \mathrm{g} / \mathrm{kg} \mathrm{P0,75} \\
r=-0,66 * *\end{array}$ & $\begin{array}{c}-0,060 \mathrm{mn} / \mathrm{kg} \mathrm{MS} \\
\text { par g ingéré } \\
r=-0,86^{* *}\end{array}$ \\
\hline $\begin{array}{l}\text { Durée d'ingestion } \\
\text { par g/kg } \mathrm{P}^{0,75}\end{array}$ & Pas de liaison & $\begin{array}{c}-0,068 \\
r=-0,59^{*}\end{array}$ & $\begin{array}{c}-0,034 \mathrm{mn} / \mathrm{g} / \mathrm{kg} \mathrm{P}^{0,75} \\
\text { par g ingéré/kg } \mathrm{P}^{0,75} \\
r=-0,86^{* *}\end{array}$ \\
\hline $\begin{array}{l}\text { Durée de rumination } \\
\text { par jour }\end{array}$ & Pas de liaison & $\begin{array}{c}+6 \mathrm{mn} \text { par g ingéré } \\
\quad=+0,69^{* *}\end{array}$ & $\begin{array}{c}+4,99 \mathrm{mn} / \mathrm{g} / \mathrm{kg} \mathrm{p}^{0,75} \\
+0,226 \mathrm{mn} / \mathrm{g} \text { ingéré } \\
r=+0,93^{* *}\end{array}$ \\
\hline $\begin{array}{c}\text { Durée de rumination } \\
\text { par } \mathrm{kg} \text { MS }\end{array}$ & $\begin{array}{c}-5,66 \mathrm{mn} / \mathrm{kg} \mathrm{MS} \\
\operatorname{par} \mathrm{kg} \mathrm{PV} \\
=-0,86^{* *}\end{array}$ & $\begin{array}{c}-8,35 \mathrm{mn} / \mathrm{kg} \mathrm{MS} \\
\operatorname{par} \mathrm{g} / \mathrm{kg} \mathrm{P^{0,75 }} \\
\gamma=-0,51^{*}\end{array}$ & $\begin{array}{c}-0,183 \mathrm{mn} / \mathrm{kg} \mathrm{MS} \\
\text { par g ingéré } \\
r=-0,91 *\end{array}$ \\
\hline $\begin{array}{l}\text { Durée de rumination } \\
\text { par } \mathrm{g} / \mathrm{kg} \mathrm{P}^{0,75}\end{array}$ & Pas de liaison & $\begin{array}{c}-0,069 \\
r=0,495\end{array}$ & $\begin{array}{c}-0,082 \mathrm{mn} / \mathrm{g} / \mathrm{kg} \mathrm{P}^{\mathbf{0 , 7 5}} \\
\text { par g ingéré/kg } \mathrm{P}^{\mathbf{0 , 7 5}} \\
r=-0,87^{*}\end{array}$ \\
\hline $\begin{array}{l}\text { Durée de mastication } \\
\text { par jour }\end{array}$ & Pas de liaison & $\begin{array}{c}+7,17 \mathrm{mn} \text { par } \mathrm{g} \\
\text { ingéré } \\
r=0,64 *\end{array}$ & $\begin{array}{c}+8,28 \mathrm{mn} / \mathrm{g} / \mathrm{kg} \mathrm{P}^{0,75} \\
+0,379 \mathrm{mn} / \mathrm{g} \text { ingéré } \\
r=0,96^{* *}\end{array}$ \\
\hline $\begin{array}{c}\text { Durée de mastication } \\
\text { par } \mathrm{kg} \text { MS }\end{array}$ & $\begin{array}{c}-8,75 \mathrm{mn} / \mathrm{kg} \mathrm{MS} \\
\text { par } \mathrm{kg} \mathrm{PV} \\
r=-0,87^{* *}\end{array}$ & $\begin{array}{c}-14 \mathrm{mn} / \mathrm{kg} \text { MS } \\
\text { par } \mathrm{g} \text { ingéré } \\
r=-0,59^{*}\end{array}$ & $\begin{array}{c}-0,246 \mathrm{mn} / \mathrm{kg} \mathrm{MS} \\
\text { par g ingéré } \\
r=-0,92 * *\end{array}$ \\
\hline $\begin{array}{l}\text { Durée de mastication } \\
\text { par } \mathrm{g} / \mathrm{kg} \mathrm{P} \mathbf{0 , 7 5}\end{array}$ & Pas de liaison & $\begin{array}{c}-0,137 \\
r=-0,65 * *\end{array}$ & $\begin{array}{c}-0,117 \mathrm{mn} / \mathrm{g} / \mathrm{kg} \mathrm{P0,75} \\
\text { par g ingéré } / \mathrm{kg} \mathrm{P} \mathrm{P}^{\mathbf{0 , 7 5}} \\
\boldsymbol{r}=-0,93^{* *}\end{array}$ \\
\hline
\end{tabular}

N. B. : Les liaisons sont exprimées par les coefficients de régression et de corrélation.

* $\mathrm{P}<0,05$. ** $\mathrm{P}<0,01$.

\section{Durée unitaire de rumination (DUR)}

Les variations de la durée unitaire de rumination (temps passé à ruminer par $\mathrm{kg}$ de matière sèche ingéré) sont significatives d'un jour à l'autre et d'un mouton à l'autre. 
En effet, pour un mouton donné la durée unitaire de rumination diminue lorsque son niveau d'ingestion augmente $\left(r=-0,9 \mathrm{r}^{*}\right)$.

D'autre part, d'un mouton à l'autre, cette durée est d'autant plus courte que le niveau d'ingestion en $\mathrm{g} / \mathrm{kg} \mathrm{P} \mathrm{P}^{:, 75}$ est plus élevé $\left(r=-0,5 \mathrm{I}^{*} ; n=\mathrm{I} 7\right)$ et surtout que le poids vif augmente $\left(r=-0,86^{* *} ; n=\mathrm{I} 7\right)$.

E,n faisant la même correction que pour la durée unitaire d'ingestion, nous obtenons un paramètre (durée de rumination $/ \mathrm{g} / \mathrm{kg} \mathrm{P}^{3,75}$ ) qui est indépendant du poids de l'animal $(r=0,043 ; n=I 7)$ mais qui reste lié aux quantités ingérées pour un même mouton $\left(r=\ldots 0,89^{*}\right)$ ou pour des moutons différents $\left(r=-0,49^{*}\right)$.

La durée d'ingestion par $\mathrm{g}$ de matière sèche ingérée par $\mathrm{kg}$ de poids métabolique variant elle-même en sens inverse du niveau d'ingestion, il n'est pas étonnant que les durée unitaires de rumination et d'ingestion soient liées :

$\mathrm{DUR}=7,32+0,492 \mathrm{DUI}(n=\mathrm{I} 7$, le coefficient de corrélation $r=0,4 \mathrm{I}$ n'étant cependant significatif qu'à la probabilité 0, Io).

La liaison est encore positive si on classe les données par mouton suivant le niveau d'ingestion :

DUR $=0,913+\mathrm{I}, 78 \mathrm{I}$ DUI $(n=6 ; r=0,74)$. Dans ce cas également, la liaison n'est significative qu'à la probabilité $0, \mathbf{I} 0$.

Par contre, si on n'examine que les variations des durées unitaires d'un jour à l'autre, on obtient la relation :

$$
\mathrm{DUR}=\mathrm{I} 5,63-\mathrm{I}, \mathrm{I} 78 \mathrm{DUI}(n=6 ; r=-0,77) \text {. }
$$

Dans ce cas, les variations ont lieu dans le sens contraire (significatif au seuil de Io p. IOo). Cela semble contradictoire avec les liaisons précédentes, mais peut s'expliquer par le fait que d'un jour à l'autre, chaque mouton ne réagit pas comme le groupe et que ces réactions se compensent au niveau des quantités ingérées et entre les durées d'ingestion et de rumination.

\section{Durée journalière de mastication (DM $=\mathrm{DI}+\mathrm{DR})$}

La durée journalière de mastication du fourrage a varié de manière significative d'un jour à l'autre et d'un animal à l'autre.

En effet, d'un jour à l'autre la durée de mastication a augmenté en même temps que les quantités ingérées $\left(r=0,92^{* *}\right)$. Cette liaison est encore plus étroite si on classe les données selon le niveau d'ingestion ( $x$ en $\mathrm{g} / \mathrm{j}$ ) pour un même mouton $\left(r=0,96^{* *}\right)$.

D'un animal à l'autre, la durée journalière de mastication a été indépendante du poids vif $(r=0,46, \mathrm{NS})$ mais a été d'autant plus élevée que l'appétit ( $x$ en $\mathrm{g} / \mathrm{kg}$ $\left.\mathbf{P}^{3,75}\right)$ a été plus élevé $\left(r=0,64^{* *}\right)$.

\section{Durée unitaire de mastication (DUM)}

Pour l'ensemble des moutons, le temps passé à mastiquer par $\mathrm{kg}$ de matière sèche ingérée (DUM) ne varie pas d'un jour à l'autre, d'où la liaison négative observée précédemment entre les durées unitaires d'ingestion et de rumination. Par contre, ce temps diminue pour le même mouton, lorsque les quantités qu'il ingère augmentent $(x$ en $g / j)\left(r=-0,92^{* *}\right)$; cela explique les liaisons positives entre les durées unitaires d'ingestion et de rumination lorsque les quantités ingérées varient. 


\section{Précision des paramètres}

A partir de nos données, nous pouvons estimer le nombre de moutons ou de jours nécessaires pour obtenir la valeur d'un paramètre avec une précision déterminée. Les résultats sont rapportés au tableau 4 et montrent que la rumination et la mastication peuvent être évaluées avec une bien meilleure précision que l'ingestion.

\section{TABLEAU 4}

Nombre d'animaux et de jours pour estimer la moyenne des paramètres étudiés avec des précision diverses au seuil 95 p.100

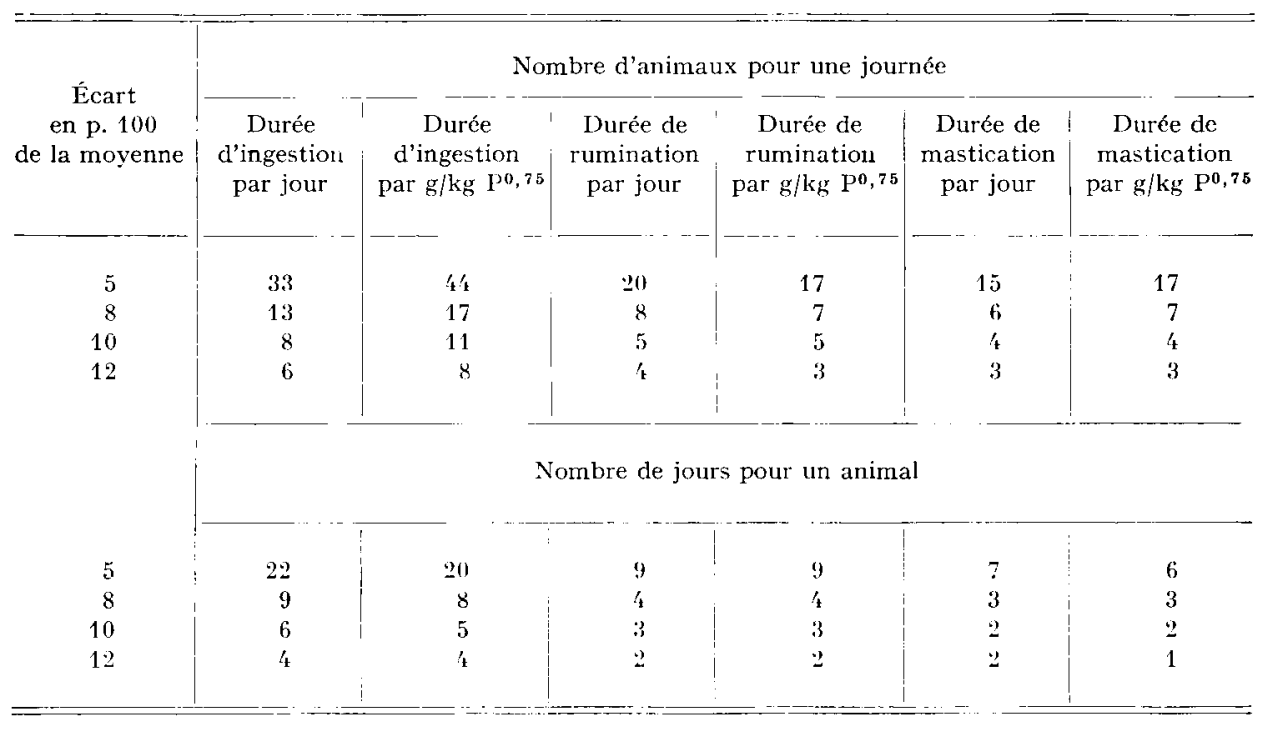

\section{DISCUSSION}

Nous avons mis en évidence l'influence de deux facteurs principaux sur le comportement alimentaire des moutons recevant à volonté un fourrage donné : le poids vif et le niveau de consommation.

Tout d'abord pour un mouton donné, alimenté à volonté, il existe des liaisons étroites entre les quantités ingérées et les différents paramètres comportementaux : c'est ainsi que les durées journalières d'ingestion et de rumination varient dans le même sens que les quantités ingérées alors que les durées unitaires d'ingestion et de rumination (en minutes $\mathrm{g} / \mathrm{kg} \mathrm{P}^{3,75}$ ) varient en sens inverse. Les variations journalières de la quantité ingérée par un animal ont vraisemblablement deux origines : elles peuvent résulter soit des variations d'un jour à 1'autre de la sensation de faim de 1'animal, soit des variations des qualités organoleptiques ou de la préhensibilité đu fourrage. Ces variations demeurent cependant faibles.

Ensuite, d'un mouton à l'autre, il existe des différences de comportement liées 
aux variations de poids vif et d'appétit entre les animaux. Les durées journalières d'ingestion ou de rumination sont indépendantes du poids vif des animaux. En revanche, plus ces derniers sont lourds moins ils mettent de temps à ingérer, à ruminer et donc à mastiquer par $\mathrm{kg}$ de matière sèche ingérée vraisemblablement parce qu'ils peuvent " traiter " plus de matière sèche par coup de mâchoire. De même qu'il est nécessaire d'exprimer les quantités ingérées en $\mathrm{g} / \mathrm{kg} \mathrm{P}^{3,75}$ si on veut les rendre indépendantes du poids vif des animaux ayant servi aux mesures, đe même il sera nécessaire d'exprimer les durées unitaires d'ingestion, de rumination et de mastication en temps passé par $\mathrm{g}$ ingéré par $\mathrm{kg} \mathrm{P}^{3,75} \mathrm{si}$ on veut les rendre indépendantes du poids vif des animaux. Quoique exprimées en $\mathrm{g} / \mathrm{kg} \mathrm{P}^{3,75}$ les quantités ingérées varient d'un animal à l'autre et traduisent des différences intrinsèques d'appétit. Alors que la durée journalière d'ingestion est indépenđante de l'appétit des animaux et la durée journalière de rumination est d'autant plus longue et les durées unitaires d'ingestion, de rumination sont d'autant plus courtes que l'appétit des animaux est plus élevé.

Il s'agit maintenant de savoir dans quelle mesure les variations des paramètres du comportement alimentaire pour un mouton donné ou entre moutons sont les conséquences des variations des quantités ingérées qui pourraient alors varier autour d'une moyenne fixée par des phénomènes plus généraux réglant l'appétit ou si elles en sont en partie les causes?

La durée journalière d'ingestion semble n'être qu'une conséquence de la quantité ingérée. En effet lorsque les moutons reçoivent pour la première fois un fourrage d'appétibilité médiocre ils mangent plus lentement mais augmentent leur temps d'ingestion pour en ingérer suffisamment (DUL PHy, résultats non publiés). D'autre part, si on enlève les bols alimentaires avant leur arrivée dans le rumen (CAmpling, BAL,CH, I96I) la durée d'ingestion de l'aliment par les animaux augmente considérablement. Enfin d'un animal à l'autre, la durée d'ingestion varie peu et les différences de quantités ingérées sont liées essentiellement à des différences de vitesse d'ingestion. Il est, par contre, plus difficile de savoir si les variations de la vitesse d'ingestion sont la cause ou la conséquence des variations des quantités ingérées. Nous pouvons dire simplement que la vitesse d'ingestion doit diminuer quand la sensation de faim diminue et c'est d'ailleurs ce qui se produit au cours du repas.

Qu'en est-il pour la rumination? On sait que celle-ci est nécessaire pour accélérer la réduction des fourrages en particules suffisamment fines pour franchir l'orifice réticulo-omasal et par là, pour quitter le rumen. Dans certains cas la rumination est peut-être un facteur limitant de la quantité ingérée. C'est ainsi que PEARce et MOIR (I964) en empêchant avec une muselière la rumination et WELCH (I967) en la rendant moins efficace par l'introduction dans le rumen de fils de nylon ont provoqué une réduction très importante de la quantité volontairement ingérée par le mouton. Cependant dans notre étude la rumination apparaît plus comme un phénomène passif influencé par les quantités ingérées et cela pour le même animal, ou d'un animal à l'autre. De même pour des fourrages verts de digestibilité élevée et qui sont digérés rapidement la durée de rumination n'est pas un facteur limitant car elle augmente quand la digestibilité diminue (DEMARQUILLY, résultats non publiés) ; elle le devient peut-être lorsque la digestibilité diminue au-dessous d'un certain niveau et que le fourrage se digère lentement.

En définitive ces résultats nous permettent tout d'abord de mieux analyser les variations du comportement alimentaire des moutons d'un jour à l'autre et d'un animal 
à l'autre. Ils nous permettent également de poser le problème des liaisons entre les quantités ingérées et le comportement. Des analyses plus précises devront être poursuivies pour faire la part des phénomènes passifs et des phénomènes actifs pouvant nous fournir des indications intéressantes sur les facteurs réglant 1'appétit pour les fourrages.

Reçu pour publication en mai 1971.

\section{SUMMARY}

\section{INFLUENCE OF LIVE WEIGH'T \\ AND FOOD INTAKE LEVEL, UPON THE FEEDING BEHAVIOUR \\ AND RUMINATION IN SHEEP}

I. The influence of live weight and food intake level upon the feeding behaviour and rumination was studied in $I 7$ sheep receiving the same green forage (2nd brome cycle) during 6 days.

2. This study shows that the live weight of the animals does not affect the daily times spent eating and ruminating. On the other hand, when the live weight increases, the dry matter $(\mathrm{g} / \mathrm{day})$ increases and the rates of eating and ruminating (daily time spent eating and ruminating corrected for $\mathrm{kg}$ dry matter intake) decrease. However, the dry matter intake expressed in $\mathrm{g} / \mathrm{kg} \mathrm{P}^{\mathbf{0 . 7 5}}$ and the rates of eating and ruminating expressed in minutes $/ \mathrm{g}$ ingested $/ \mathrm{kg} \mathrm{P}^{0.75}$ are independant of the live weight of the animals.

3. From one sheep to another, the food intake (in $\mathrm{g} / \mathrm{kg} \mathrm{P}^{\mathbf{0 . 7 5}}$ ) is in a positive correlation with the daily rumination time and in negative correlation with the rates of eating and ruminating ; under these conditions the daily eating time does not vary.

4. From one day to another, when the food intake per sheep increases, the daily times spent eating and ruminating increase whereas the rates of eating and ruminating $\left(\mathrm{min} / \mathrm{g} / \mathrm{kg} \mathrm{P}^{0.75}\right)$ decrease.

5. The relationships between the behaviour of the animals and their food intake are discussed. Thus, the daily eating time only seems to be a consequence of the amount of food ingested. On the other hand, it is difficult to say if the variations of the eating rate are the cause or the consequence of the variations of dry matter intake. In this trial, rumination seems to be a passive phenomenon influenced by the amounts of food ingested.

\section{RÉFÉRENCES BIBLIOGRAPHIQUES}

CAmpling R. C., Balch C. C., I96I. Factors affecting the voluntary intake of food by cows. Brit. J. Nutr., 15, 1523 .

Demarouilly C., Weiss Ph. 1970. Tableaux de la valeur alimentaire des fourrages. Étude S.E.I., no ${ }^{\circ 2}$.

Gordon J. G., I965. The relationship between rumination and the amount of roughage eaten by sheep. J. Agr. Sci. 64, I 5 I-I 55 .

Gordon J. G., I969. Rumination and its sigrificance. World review Nutrition and dietetics, 9, 25 I-273.

KERBAA M., I969. Étude de quelques facteurs de variation du comportement alimentaire et mérycique des ruminants. Thèse Doct. Sci. Université Clermont-Ferrand.

KLeIBer M., I964. Proceedings of the 3rd Symposium held at Troon on Energy metabolism, Mai I964 : Metabolic Body Siza.

Pearce G. R., Molr R. J., I964. I. The influence of rumination and grinding upon the passage and digestion of food. Aust. J. Agr. Res. 15, 635.

Ruckebusch Y., ro63. Recherches sur la régulation centrale du comportement alimentaire chez les ruminants. Thèse Doct. Sci. Université de Lyon.

WeLCH J. G., I967. Appetite control in sheep by indigestible fibers. J. Anim. Sci., 26, 849.

Welch J. G., Smith A. M., Gibson K. S., I97o. Rumination time in four breeds of dairy cattle. J. Dairy. Sci., 53,89.

Welch J. G., Smith A. M., I969. Effect of varying amounts of forage intake on rumination. J. Anim. Sci., 28, 827. 